\title{
Requirements and Technologies Towards UAM: Communication, Navigation, and Surveillance
}

\author{
M. Cenk Ertürk, Honeywell Aerospace, Redmond, WA. \\ Nozhan Hosseini, Hosseinali Jamal, Alphan Şahin, David Matolak, University of South Carolina, Columbia, SC. \\ Jamal Haque, University of South Florida, Tampa, FL.
}

\section{Abstract}

Urban air mobility (UAM) is a concept for creating an airborne transportation system that operates in urban settings with an on-board pilot and/or remote pilot in command (RPIC), or with a fully autonomous architecture. Although the passenger traffic will be mostly in and near urban environments, UAM is also being considered for air cargo, perhaps between cities. Such capability is pushing the current communication, navigation and surveillance (CNS) / air traffic management (ATM) systems that were not designed to support these types of aviation scenarios. The UAM aircraft will be operating in a congested environment, where CNS and ATM systems need to provide integrity, robustness, security, and very high availability for safety of UAM operations while evolving. As UAM is under research by academia and government agencies, the industry is driving technology towards aircraft prototypes. Critical UAM requirements are derived from command and control (C2) (particularly for RPIC scenario), data connectivity for passengers and flight systems, unmanned aircraft systems (UAS) to UAS communication to avoid collision, and data exchange for positioning and surveillance. In this paper, we study connectivity challenges and present requirements towards a robust UAM architecture through its concept of operations. In addition, we review the existing/potential CNS technologies towards UAM, i.e., 3rd generation partnership project (3GPP) fifth generation $(5 \mathrm{G})$ new radio (NR), navigation detect \& avoid (DAA), and satellite systems and present conclusions on a future road-map for UAM CNS architecture.

Keywords: 5G, CNS, DAA, NASA, requirement analysis, radio technical commission for aeronautics (RTCA), satellite systems, UAM.

\section{1- Introduction}

NASA's UAM represents one of the most ambitious challenges for the aviation industry. With the goal of ultra-safe and efficient aircraft movement of passengers and freight near and within urban environments, this operation will require advances in a range of engineering domains, including propulsion, airframe design, ATM and CNS.

A NASA UAM market study [1] defined three most challenging UAM use cases as, last-mile delivery, air metro, and air taxi, for possible implementation by 2030. NASA's UAS traffic management (UTM) program has been operating for a few years, progressively advancing use of UAS operating at very low altitudes (e.g. below 400 feet, sometimes termed very low-level (VLL) air-space/operations). UTM is planned to provide a set of traffic management services via a federated group of UTM Service Suppliers, comparable to traditional air traffic control (ATC) services provided to visual flight rules (VFR) and instrument flight rules (IFR) aircraft.

Rapid delivery of packages $(<5 \mathrm{lb})$ from a local depot hub to a customer or next depot represents so-called last mile delivery. At predetermined schedules, routes and stops throughout a city are envisioned, similar to bus or metro routes on the ground. An air taxi scheme (door to door) ride-sharing operation with customer defined drop-off and destination depots is another UAM application. All three of these applications require sophisticated route planning and accurate navigation. An efficient DAA system is also required for UAM operations and planning.

The focus of this paper is to understand the connectivity challenges and determine the requirements toward robust UAM through its concept of operations. Once the baseline scenario and its requirements are established, we investigate the existing/potential CNS technologies towards UAM, i.e., 3GPP 5G NR, navigation DAA, and satellite systems, and provide a potential road-map for UAM CNS architecture.

The rest of this paper is organized as follows. In Section 2 , we define requirements for UAM scenarios that addresses the potential connectivity challenges. Section 3 presents the NASA and RTCA activities in terms of programs, standards and concept of operations (Con-Ops). Section 4 investigates $5 \mathrm{G}$ capabilities and studies from the UAM application and requirements point of view. Precise navigation systems and accurate DAA systems, which are considered one of the key technology enablers of UAM, are studied in Section 5. In Section 6, we investigated the satellite support for beyond lineof-sight (BLOS) scenarios (or multi-link support scenarios). Specifically, low-earth orbit (LEO) satellites are presented as they have a potential of high data rate and low latency support for terrestrial platforms. Conclusions and potential study items are presented in Section 7.

\section{2- UAM Connectivity Challenges and Requirements}

UAM transportation is aiming to provide services for airborne passengers, and cargo applications. The ideal world 
would be an autonomous vehicle that could reliably provide the service, including assurance of safe operation for humans on board. The system requirements need to consider the challenges and differences between urban and rural environments, i.e., dense urban settings like New York city etc. These kinds of urban environments imply greater challenges than in existing aviation, given the density of UAM aircraft will be higher, and line-of-sight (LOS), non-line-of-sight (NLOS), and BLOS links will experience intermittent blockages. In addition, any navigation signaling will also be stressed in terms of accuracy and latency. Given the likes of terrestrial services such as UBER, Lyft and others, the application that is drawing the most attention is the concept of air taxi. This in turn is driven by the recent advances in autonomous vehicles, lead by Tesla, which include battery technology, battery powered propulsion systems, and communication and navigation systems. The success of such services will highly depend on system robustness and safe operations.

Several, if not all, elements of UAM are under investigation: propulsion systems, flight control systems, precise and rapid navigation and surveillance, emergency landing systems and terrain mapping, use of artificial intelligence (AI) for enhanced flight safety, off loading processing using cloud services, C2 and non-command and control (nC2) (sometimes referred as payload) communications, detect, sense, and avoid, and related sensors. Given the operational reliability of these sub-systems, the possibility of unmanned UAM vehicles managed by a RPIC is also being considered. For such a case, the communication systems must be extremely robust, secure, always available and should satisfy a high level of data integrity. While independently some or most of these systems do exist, the need for low size, weight, power, and cost to meet the business targets are key drivers.

\section{A. Data Types: $C 2$ and non-C2}

The definition of $\mathrm{C} 2$ data is all information that is involved with flight controls or safety systems. This is also evolving given how much of flight system diagnostics data will be needed in real-time, to assess flight operation for possible abort. Example AI operating across cloud services could be critical to flight operation, the use of live images to assist remote operation, etc. All other data will fall into the $\mathrm{nC} 2$ category, i.e., downloading of post flight data, passenger information etc. Thus the requirements need to consider these challenges to provide such connectivity for control and nonpayload communication (CNPC), also known as C2 communication. In addition, the functions of CNPC can address different types of information such as telecommand messages, non-payload telemetry data, support for navigation aids, ATC voice relay, air traffic services data relay, target track data, airborne weather radar downlink (DL) data, non-payload video DL data, etc. The $\mathrm{C} 2$ communication link should also support secure and reliable communications between the RPIC and the aircraft to ensure safe and effective unmanned air vehicle (UAV) flight operation.
Relative to $\mathrm{C} 2$, the payload communication link $\mathrm{nC} 2$ may be used for data applications that often requires high throughput. Payload communication types depend on application (e.g., agriculture, public safety, passenger on-board), and can thus vary widely. It can be assumed that disruption of payload $\mathrm{nC} 2$ links —albeit inconvenient - is not critical, whereas C2 link disruption will be critical.

\section{B. Integrity}

Given the increase in cyber-malicious activities, UAM C2 data communications will have to support several layers of data integrity and authentication. Creating a complete separate and custom communication link with protected spectrum seems like the logical path, but funding such large scale global deployment will be at a likely prohibitive cost. Thus the ability to leverage the existing commercially or crowd-funded systems is appealing. Such an integrity system will ensure messages received by the UAM nodes are authenticated and verified. Thus the evolution of commercial 5G and LEO satellites services are in the trade space. If and when the services evolve towards RPIC, requirements to support such feature must be addressed comprehensively.

\section{Spectrum and Carrier frequencies}

The highly priced and congested spectrum is the real estate of future communication systems. Thus the cellular frequency bands (sub- $6 \mathrm{GHz}$ ), 3GPP 5G NR, millimeter wave (mmWave) bands (24 GHz-86 GHz), evolving LEO satellite bands, as well as existing aviation bands are in the trade space. As we move to higher frequencies, radio signals experience large free-space and tropospheric attenuation, which will limit range. While the 5G mmWave systems will offer a higher throughput, they are mostly limited to LOS and short range, and most likely available in urban environments. The UAM system will have to resort to lower date rates as it travels to outer perimeters of cities. This is where the insertion of satellites based BLOS capability becomes viable, putting pressure for all flight services to adjust for data rates and associated delays.

\section{Robust/Reliable Communications}

One obvious difference between UAM and traditional aviation settings is the proximity of aircraft to obstacles, due to the low altitude of near-urban and within-urban environments. This poses a propagation challenge, typically termed obstruction or blockage in the satellite community, and shadowing in the terrestrial communications community. Attenuation due to such blockages can be several tens of $\mathrm{dB}$, which can be enough to sever a link. Thus connectivity between aircraft and C2 stations will most likely be accomplished with multiple links.

For LOS links, air-ground connectivity will generally be preferred, because of its power efficiency and low latency in comparison to satellite or high altitude platform system (HAPS) links. The term BLOS is used here to mean a link that is beyond the reach of a ground station (GS), but can 
be reached via other means, such as other airborne platforms or satellites. The term NLOS is used to mean a link where no LOS exists between the aircraft and any station, ground, airborne, or satellite. The NLOS case is typically the most challenging, from both the power and channel distortion perspective (due to multipath components, MPCs). Once UAM is fully deployed, NLOS cases should also be the most rare.

From the PHY and data link perspective, the primary differences between $\mathrm{C} 2$ and $\mathrm{nC} 2$ links are as follows:

- C2 links require extremely high reliability and availability, typically availability of $99.999 \%$ or larger. Reliability/availability requirements for $\mathrm{nC} 2$ links vary with application, but will not be as stringent.

- Although not all requirements have been defined, most C2 link data rates will be moderate, e.g., $<300 \mathrm{kbps}$ for compressed video transmission. In contrast, the $\mathrm{nC} 2$ link data rates could be orders of magnitude larger, particularly for passenger applications.

- The C2 link latency will need to be small for certain functions. This will require message or packet size to be very small as well when $\mathrm{C} 2$ link data rates are low.

At the network layer, notable points include the following:

- Some C2 transmissions will be multicast and/or broadcast (e.g., for a GPS local area augmentation system), whereas many $\mathrm{nC} 2$ links will be point-to-point.

- Some C2 transmissions may not tolerate the latency of relaying over multiple hops.

- Another consideration distinguishing the $\mathrm{C} 2$ and $\mathrm{nC} 2$ links is robustness or resilience.

In short, C2 links should be extremely tolerant of interference, and should re-establish themselves very rapidly after any disruption.

In military systems this comes under the topic of electronic warfare (EW). This includes for example,

- Jamming, which is the intentional transmission of in-band interference that degrades performance, possibly to the point of link outage;

- Control channel "flooding," which is the intentional transmission of signals over multiple access channels that prevents legitimate platforms from accessing the available channels;

- Spoofing, which is when an unauthorized entity "masquerades" as a legitimate system user or controller, and sends messages with the intent to disrupt system operation.

These three forms of EW are listed from the least sophisticated to the most sophisticated. Depending on the resources available to the disrupting entity, adapting mobile networks of such jammers/flooders/spoofers can be deployed, which could effectively disable a network of UAM links entirely.

Methods to make C2 signals robust against such disruptions include spread spectrum and multi-band signaling, signal spatial filtering, usually via beamforming and beamtracking, strong forward error correction (FEC) techniques, active interference cancelling, and strong encryption. Adaptive routing at the network layer can also enhance robustness, at the expense of some latency. No current commercial system uses all of these techniques; existing cellular systems employ a few, e.g., encryption and FEC.

\section{E. Security}

Security can be discussed in terms of system robustness to disruption, which is related to availability, discussed in the previous section. It can also be discussed in terms of accuracy of message transfer (e.g., resilience to spoofing). Having any entity receive and understand the majority of UAM C2 messages will not generally pose a problem, unless that entity uses those messages against UAM communications (e.g., via flooding or spoofing). Anti-spoofing requires strong authentication procedures.

Security issues are highly-related to regulations for UAVs. The federal aviation administration (FAA) stated remote identification (remote ID) of UAS, crucial to integration efforts [2]. As defined by FAA, remote ID is the ability of a UAS in flight to provide identification information that can be received by other parties. This adds more regulations, which could assist the FAA, law enforcement, and other organizations including federal agencies to track UAVs, and recognize as FAA states "unsafe manner" UAS, including those not allowed to fly. According to [2], the new rules would facilitate the collection and storage of certain data such as identity, location, and altitude regarding UAVs as well as its control station. In June 2017, FAA charted a comprehensive report, UAS identification and tracking aviation rulemaking committee (ARC) [3] related to the remote ID, and mentioned technologies available to identify and track drones in flight and other associated issues. According to [3], among viable technologies mentioned are automatic dependent surveillance-broadcast (ADS-B), lowpower direct RF (such as Bluetooth, Wi-Fi, RFID), cellular systems, satellite, software-based flight notification with telemetry, unlicensed integrated C2, physical indicator, and visual light encoding. In tables provided in [3], there are analysis and comparisons of these viable technology solutions in terms of different factors such as ease of compliance for owner/operator, performance against requirements, security, inter-operability, costs, and, etc. Although, the FAA has indicated that any solution for remote ID and tracking should avoid causing interference on the FAA's secondary surveillance radar (SSR), airborne collision avoidance systems (ACAS), and ADS-B systems. Therefore, it is highly recommended that any proposal for using ADS-B frequencies must be thoroughly investigated.

As mentioned in [3] this new tracking system is also in the same direction as ATC, or possibly ATM in aviation. ATC as described by FAA [4], has the primary responsibility of the separation of the aircraft. Controllers from traffic controllers on the airport speak directly with pilots, notifying them of traffic or weather in their vicinity, while ATM as a whole managing system facilitate the approach to managing traffic that considers the impact of individual actions. There are many factors, for example bad weather, traffic overloads, or 
emergencies that requires consideration of who or what may be impacted by events, and a coordinated mitigation effort to ensure safety, efficiency and equity in the delivery of air traffic services. Without a coordinated management, flight delays due to small disruptions can quickly expand across the country, causing flight cancellations, and significant delays [4]. According to [3] (the reader is referred to Section 6.6), there are recommendations on how ATC could inter-operate and maximize the benefits of ID and tracking. ATC operations at low altitudes especially near the major airports are often complex. As a result, ATC does not have the capabilities to track and manage all UAS operations, especially smaller UAS operating at low altitudes. Therefore, the ARC recommends the UAS ID and tracking system should inter-operate with the ATC automation, such that target information from the ID and tracking ground system, including ID and position, can be send to ATC.

\section{F. Communication Zones}

UAM aircraft requiring links with larger data rates and longer communication distances bring new research dimensions for future aeronautical communication systems:

- Power efficiency and spectral efficiency are not usually equally important for UAM scenarios as compared to terrestrial communications. The aviation communication zones are typically larger than in terrestrial communication systems, in order to achieve longer distances. Hence, power is considered to be much more precious than bandwidth.

- In aeronautical stations, power must be rigorously conserved since all of it is supplied on-board and must be shared among many power consuming systems.

- Aeronautical channels can often be modeled as Ricean fading channels, where the Rice factor (i.e., $K$-factor) can span a range between best case additive white Gaussian noise (AWGN) to a near-worst case Rayleigh fading channel.

\section{G. Availability}

The cellular industry is also interested in using UAVs to expand their capacity and revenue to provide cost effective wireless connectivity for devices without coverage by the existing infrastructure. Additional cellular applications, e.g., as user equipment or relays, are also likely.

\section{H. Network Topology: Distributed or Centralized}

Multiple links can be provided in centralized coordinated multi-point transmission and reception schemes through 5Glike techniques and systems. Multiple links can also be provided by mobile ad-hoc networks; these generally require more advanced procedures at the network layer, e.g., adaptive routing. A third option for multiple links can be satellite communications or high-altitude platforms (HAPS).

\section{Date rate/Bandwidth}

Data rates for $\mathrm{C} 2$ links are expected to be modest (e.g., a maximum of $300 \mathrm{kbps}$ for compressed video, which would not be used continuously). In contrast, the payload communication (nC2) link is usually used for data applications, and often requires high throughput. Passengers on UAM aircraft will desire (or require) data rates similar data to those that they obtain in terrestrial networks.

\section{J. Air-Interface Design Considerations}

Power efficiency is of critical importance in a UAM scenario, compared to emphasis on spectral efficiency in terrestrial networks. As cell sizes increase, the amount of transmit power amplifier (PA) back-off becomes a very critical issue. For UAM scenarios, power-efficient constant-envelope modulation schemes may need to be considered.

One critical technical drawback of new generation aeronautical waveforms (L-band digital aeronautical communication system (LDACS), aeronautical mobile airport communication system (AeroMACS), i.e., in general multicarrier (i.e., orthogonal frequency division multiplexing (OFDM)like) schemes) is that these waveforms have a high peak-toaverage ratio (PAPR). Therefore, in order not to overdrive the PA, a back-off of around $12-18 \mathrm{~dB}$ is needed from the maximum output power of the PA. In a one-way communication link-budget, this corresponds to a range degradation: e.g., $12 \mathrm{~dB}$ back-off means a factor of 4 smaller range; $18 \mathrm{~dB}$ backoff means a factor of 8 smaller range. This is in comparison to traditional single-carrier schemes such as the VHF digital link (VDL) waveforms. Roughly, cell sizes around $300 \mathrm{~km}$ in VDL reduce to $37.5 \mathrm{~km}$ to $75 \mathrm{~km}$.

On the other hand, VDL-like waveforms have a drawback in terms of limited bandwidth (data-rate) (i.e., VDL Mode 2 or Mode 3 bandwidth is $25 \mathrm{kHz}$ ). The aeronautical community is more cautious about moving to OFDM-based waveforms as the communication ranges are larger in aeronautical scenarios and deployment costs will increase dramatically (as more cells will be needed to cover a given area). Yet, similar problems in the power requirements of terrestrial cellular have been studied and special waveforms (i.e., discrete Fourier transform-spread orthogonal frequency division multiplexing (DFT-s-OFDM)) that decrease back-off are already being investigated in the cellular communications community. The use of multicarrier schemes was primarily driven by two reasons: their relatively simple equalization for dispersive terrestrial channels, and the medium access flexibility provided by joint time-frequency partitioning. Aeronautical channels with strong LOS will not experience significant dispersion for short-range UAM links, but the flexibility of multicarrier schemes is still desirable. In addition, even if range for some UAM links is short, the small platform size of some aircraft will still translate to powerlimited communication links.

As VDL signals have near constant envelope by virtue of their single carrier design, and hardware presently exists to accommodate preliminary phases of UAM, the aeronautical community may employ VDL type waveforms for the 
initial C2 applications. As the market grows and additional requirements become important, the UAM community should consider new waveforms, perhaps with the support of the $5 \mathrm{G}$ community. These waveforms should provide some multipath resilience for a Ricean aeronautical channel and a low PAPR, while maintaining availability, security, integrity, and data rate requirements. The developments related to $3 \mathrm{GPP} 5 \mathrm{G}$ NR for non-terrestrial networks (NTN) are summarized in Section 4.

\section{3- UAM Programs: NASA and RTCA Activities}

The UAM Con-Ops will notionally consist of the following:

- Shared airspace: UAM traffic will coexist with other aircraft.

- UTM: When more than one vehicle operates within a defined airspace, a traffic management system must be established to manage the airspace and disseminate realtime situational awareness information to RPICs and/or pilots on board, or between the on-board flight management systems of aircraft.

- Air corridors: In city centers UAM nodes will be required to travel in a designated air highway or tunnels. Here, an effective "box" or "lane" of some height and width will be designed with speed limits and rules for the air highway.

- Designated takeoff/landing sites: UAM operations will only use fixed departure and arrival air terminals, with equipment service capability and passenger loading and unloading.

- Vertical takeoff and landing (VTOL) only: It is likely that only vertical takeoffs and landing will be allowed in the city-center, via stations often referred to as vertiports.

\section{A. NASA Activities}

NASA launched an UAM Grand Challenge Program and the concept is illustrated in Fig. 11 This is a four-year testing program on unmanned aircraft integration, and beginning in 2020, NASA will start field trials in urban environments with select participants, evaluating all subsystems of UAM operations under a variety of weather, traffic and contingency conditions. The objective of this program is to establish an understanding of different systems and the performance required to achieve a mature UAM system capable of operating efficiently in dense urban environments. During these test trials, NASA and government partners will conduct tests and collect data on various phases of UAM operation to help determine vehicle certification requirements. Testing will be focused on UAM with passenger carrying capacities, with vertical takeoff and landing and potentially standard takeoff and landing.

\section{B. RTCA Activities}

In USA a standard has been adopted for CNPC, created by the RTCA [5]. This standard specifically pertains to the L-band (900-1000 MHz) and a portion of C-band allocated to aviation (5.03-5.091 GHz). The standard applies to air-toground (AG) links LOS only, and the committee is at work on the BLOS standard. Estimated UAV CNPC bandwidth

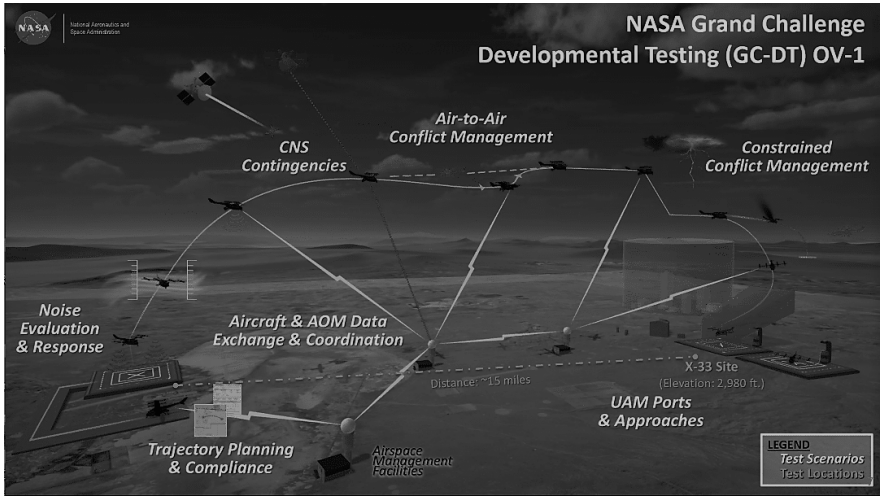

Figure 1. NASA Grand Challenge: Definitions and Scenarios [1]

requirements for the year 2030 are $34 \mathrm{MHz}$ for the terrestrialbased LOS CNPC, and $56 \mathrm{MHz}$ for the satellite based BLOS CNPC link [6]. The RTCA standard does not specify any 5G applications, and primarily addresses the three lowest layers of the communications protocol stack. The standard is though general enough to be used for any type of $5 \mathrm{G}$ application involving medium and large sized UAVs. In the United States, UAS CNPC deployment is planned in two phases, in which Phase 1 supports terrestrial networks (based on proprietary handover functionality) but does not address any industry standard handover capability, which will be addressed in phase 2. The frequency bands allocated for CNPC Phase 1 are Lband and C-band. For future CNPC BLOS CNPC, defined in Phase 2, satellite communications using $\mathrm{L}, \mathrm{C}, \mathrm{Ku}$, or $\mathrm{Ku}$ bands, as well as networked terrestrial and C-band terrestrial will be considered.

\section{4- 5G New Radio towards UAM}

In 3GPP 5G NR terminology [7], NTN are defined as networks, or segments of networks, using an airborne or spaceborne vehicle to employ transmission to or from a relay node or base station. The NTNs are expected to enhance the 5G NR service reliability by providing service continuity for machine-to-machine (M2M) and Internet-of-Things (IoT) devices or for passengers on board moving platforms such as aircraft. The standards are also aiming at ensuring service availability anywhere, especially for critical communications and future aeronautical communications. In 3GPP, to support NTN, the potential areas that impact the specifications, e.g., the physical channels and modulation in Release 16 [8], are identified as follows: 1-Propagation channel, 2- Frequency plan and channel bandwidth, 3- Power-limited link budget, 4Cell pattern generation, 5- Propagation delay characteristics, 6- Mobility of the infrastructure's transmission equipment, 7Service continuity between land-based 5G access and nonterrestrial based access networks, and 8- Terminal mobility. However, it is still not clear if the near-term developments related to NTN can address the requirements related to UAM or not. 
There are two major technical reports [7], [9], which have been updated based on agreements in 3GPP meetings, for $5 \mathrm{G}$ NR to support NTN. While [7] aims at collecting findings in the 3GPP RAN meetings, [9] consists of a set of necessary features/adaptations enabling the operation of the NR protocol in NTN for 3GPP Release 17 based on the observations in [7]. Several topics captured in [7] are as follows: 1) the NTN deployment scenarios and related system parameters (e.g., architecture, altitude, orbit, etc.); 2) adaptation of the 3GPP channel models for NTN (e.g., propagation conditions, mobility, etc.); and 3) the areas on the NR interface that may need further evaluations. It is worth noting that NR prioritizes satellite access and considers UAS and including HAPS as a special case of NTN with lower delay/Doppler values and variation rates [9]. It is expected that 3GPP 5G NR Release 17 supports NTN.

The 5G NR with the support of NTN aims at establishing a radio link between the user equipment (UE) and the space/airborne platform, called service link, operating in frequency bands above and below $6 \mathrm{GHz}$. The recommended deployment scenarios are based on a handheld/IoT device (23 $\mathrm{dBm}$ transmitter (TX) output power) or a very small aperture terminal, e.g., bus, train, vessel, aircraft (33 dBm TX output power + 43.2 dBi TX antenna/ $39.7 \mathrm{dBi}$ receiver $(\mathrm{RX})$ antenna gain). For the air/space borne vehicles, geostationary satellites, non-geostationary satellites with low earth orbiting satellites (from $600 \mathrm{~km}$ up to $1500 \mathrm{~km}$ ), medium earth orbiting satellites (from $7000 \mathrm{~km}$ up to $20000 \mathrm{~km}$ ), and airborne platforms (from $8 \mathrm{~km}$ to $50 \mathrm{~km}$ ) are considered. The details on the deployment scenarios are given in Table 5.3-5.1 in [7].

3GPP recommends a comprehensive channel model which includes outdoor-to-indoor penetration loss, atmospheric absorption, rain and cloud attenuation, scintillation, Faraday rotation, time-varying Doppler shift, etc. for NTN performance evaluations in $5 \mathrm{G}$ NR. It has been noted that the fading characteristics can be modeled as mostly Ricean with a strong direct signal component, but slow fading can also occur due the temporary shadowing due to the obstacles such as trees, buildings, or other structures, e.g., large bridges. On the other hand, HAPS may yield more multipath components than satellites. The NTN channel characteristics can have an impact on reference signal design, particularly for random access channels, as NTN would introduce large Doppler shifts compared to those for terrestrial networks. Larger subcarrier spacing for OFDM (i.e., smaller symbol duration) is recommended to protect against distortion due to Doppler shifts. In 5G NR R15, phase-tracking reference symbols (PTRS) was introduced, which was not available in 4G LTE [10]. It has been noted that flexible configuration of PTRS would be helpful to compensate residual carrier frequency offset and Doppler shifts. However, from the large scale perspective, supporting very high speed UEs such as aircraft systems featuring maximum speeds of $1000 \mathrm{~km} / \mathrm{h}$ is still challenging, and may require some faster power control loops than what $5 \mathrm{G}$ NR offers.

The 3GPP recognizes the benefit of satellites for critical communications including public safety communications due to their dependability and large coverage area, while accepting the issue of the propagation delay of satellite systems for certain applications requiring low latency. It has been noted the one-way delay between the UE and the RAN (on-board the satellite/HAPS or on the ground) can reach up to 272.4 ms for geostationary synchronous orbit (GSO) systems and is larger than $14.2 \mathrm{~ms}$ for non-geostationary synchronous orbit (NGSO) systems. For HAPS, the one-way delay is less than $1.6 \mathrm{~ms}$, which is comparable to cellular networks. It has been emphasized that it is likely that these delays impact all signaling loops, especially at access and transport levels.

The large coverage also creates some issues, e.g., more variations on the propagation delay. For example, one issue is that the ratio between propagation delays at cell center and cell edge is likely to be higher in the context of HAPS than geostationary satellite systems, which may alter the design of contention-based access channels (of which there may be very few in UAM links). Another issue related to the large varying delay due to the fast moving satellite/HAPS and UEs is that the timing advances of the UEs may need to be updated more dynamically. In [9], autonomous acquisition of the TA at UE with known location and satellite ephemeris or indication of common TA to all users within the coverage of the same beam with broadcasting was considered as potential solutions. The hybrid automatic repeat request (HARQ) scheme in NR and adaptive modulation and coding are also expected to be impacted because of large timing jitter values in NTN. Another issue related to propagation delay is the duplexing mode. In [7], it is recommended not to discard TDD for HAPS and LEOs (in case regulations (ITU/R and/or national) allow), although time-domain duplexing (TDD) requires guard times, which are a function of propagation delay, to avoid simultaneous transmit and receive.

The main design challenges in NR are listed as 1) achieving high throughput for power-limited link budget (e.g., for a given transmit power from the UE on the uplink (UL) and from the satellite/HAPS on the DL), and 2) availability of the service under deep fading situations (e.g., 20 and $30 \mathrm{~dB}$ in Ka band, similarly for shadowing in lower frequency bands). One way of improving throughput/power ratio is to set the operating point in the PA as close as possible to saturation point. However, this may require changes in both UL and DL channels in 5G NR. For a satellite/HAPS on the DL, OFDM that is considered for terrestrial network may not be the best solution. For example, in [7], it is emphasized that an additional $2 \mathrm{~dB}$ back-off can reduce the link capacity by $20 \%-40 \%$. Finally on this topic, we note again that UAM C2 link data rates will not be very large, so achieving high throughput is of lesser importance than link reliability and availability.

In [11] and [12], Inmarsat, Intelsat, SES and Fraunhofer IIS proposed that alternative OFDM-based schemes need to be considered in the DL, which are closer in characteristics to current state of the art single carrier (SC) schemes, such as single carrier frequency division multiple access (SC-FDMA) (i.e., DFT-s-OFDM) and variants. Although this essentially 
requires redesign of the shared channels, i.e., a major standardization impact, it may be a necessary improvement for reliable NTN given the large out-of-band emission (OBO) for OFDM waveform. In [7], it is also emphasized that extended multicarrier modulation and coding schemes for UL that features low PAPR are needed. To ensure operation at low $E_{s} / N_{0}$, alternative modulation and coding schemes and redefined physical resource blocks (e.g., single tone transmission rather than 12 subcarriers) are recommended. Note that the PAPR is a function of the number of subcarriers and of the modulation order, depending on the waveform type.

\section{5- Navigation and Detect \& Avoid}

Growth in the number of UAM nodes will demand a high-accuracy positioning system from both self awareness and situational awareness points of view. Addressing sufficiently sophisticated implementable sensor fusion and tracking algorithms to provide both accurate self-positioning and situational-awareness (what is around to avoid) has a vital importance toward reliable UAM operations.

The FAA regulations will strictly ask UAM drones to "squawk" which means to broadcast their ID and position via drone remote ID gear as well as manned aircraft ID that used in ADS-B systems and transponders. This is essential for sense and avoid systems and is the goal of FAA and industry. By 2020, all aerial vehicles should mandatory equip ADS-B "out" system which allows vehicles to broadcast their position, vector, altitude and velocity [13]. All manned aircrafts operating in airspace should use Mode $\mathrm{C}$ transponder, however, as reported in [13] regulations excluded manned aircraft without electrical systems which as reported in [13], 30 percent of crop dusters in Mississippi Delta had no electrical system. Compliance issue reported in a study by FAA, that for one example they reported just six months out from mandatory ADS-B compliance only 44 percent of general aviation had installed ADS-B out equipment. Note that FAA only mandates ADS-B out equipment and not "in". which gives aircrafts the ability to follow position, ID of other traffic targets. FAA still not decided on equipping drones with ADS-B out because of concerns of saturating the ADS-B broadcast frequency. Note that ADS-B "in" does not have a similar problem and is under consideration for small drone mounting which makes it an open topic for small drones industry to see if customers are willing to pay for drone remote ID receivers. Author in [13], claimed that the drone community and FAA regulators understand this problem and are designing UTM with drone remote ID and ADS-B "In" as an internal part of collision avoidance and sense and avoid plans.

A. Global Navigation Satellite System (GNSS) and Inertial Navigation System (INS)

As discussed in [14], one solution is to fuse all existing global navigation satellite systems (GNSSs) (e.g. Global Positioning System (GPS), Galileo Satellite Navigation (GALILEO), Quasi-Zenith Satellite System (QZSS), Globalnaya Navigatsionnaya Sputnikovaya Sistema

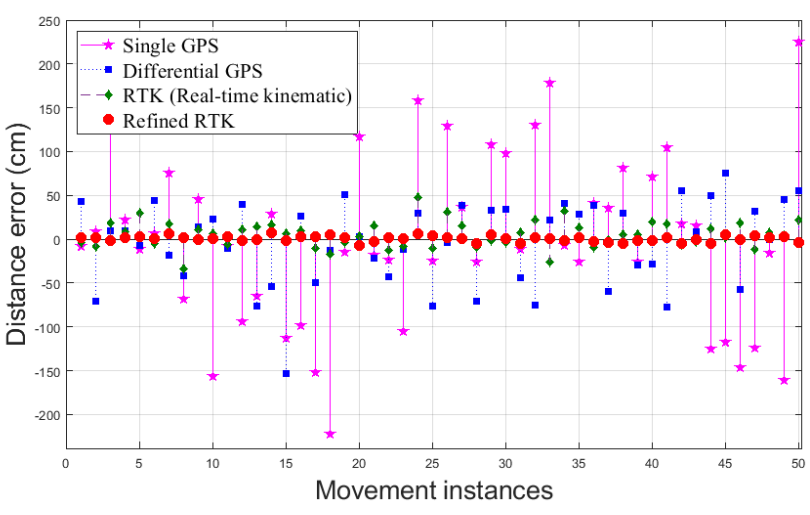

Figure 2. Comparing different positioning error distribution for different GNSSs.

(GLONASS) etc.) with improved inertial navigation systems to achieve high accuracy positioning solutions. GNSS systems have been designed for LOS environments and therefore are not so suitable for moderately dense urban areas. Based on [12], the integration of all mentioned systems will result in high accuracy, multi-band and multi-mode grade receivers whose cost are not suitable for low-cost commercial applications.

Cellular networks can also play a significant navigation role in UAM operation; specifically $5 \mathrm{G}$ implementations can provide node-to-node communication capabilities that lead to real time channel estimation and thus alternate navigation techniques. Based on [15] and [16], the distribution of measured data showed standard deviation of a single mode GPS receiver to be about $2 \mathrm{~m}$, differential GPS (DGPS) $1 \mathrm{~m}$, while cellular assisted real-time kinematic (RTK) is about $16 \mathrm{~cm}$. Novel algorithm optimization and dual antennas solution such as in [17], improved the three-dimension precision of the relative positioning of RTK under open sky condition within $2 \mathrm{~cm}$. The accuracy situation is worse for city canyons, viaducts, and tunnels, with positioning drift of $(20 \mathrm{~m}+)$ due to signal blockage and multi-path components, Also in height, when using GNSS alone. Fig. 2 shows the positioning error distribution comparison for these four systems. This positioning variance is related to an air traffic control separation, which is the name for the concept of keeping an aircraft outside a minimum distance from another aircraft to reduce the risk of collision, as well as prevent accidents due to secondary factors, such as wake turbulence.

In [18] and [19], exploiting 5G NR for $450 \mathrm{MHz}$ to $6000 \mathrm{MHz}$ and $24.25 \mathrm{GHz}$ to $52.6 \mathrm{GHz}$ transmission for high-accuracy positioning is investigated as a complementary hybrid solution to GNSS in harsh environments where an LOS path does not exist. The authors of [19] evaluated positioning accuracy of six carrierless mmWave waveforms candidates (i.e., Gaussian pulse, inverse fast Fourier transform (IFFT) pulse, Gaussian-raised cosine pulse (RCP), rectangular-RCP, sinc-RCP, and Hann-RCP) through impulse radio (IR) and compared them with multicarrier waveforms (i.e., OFDM, 
filter-bank multicarrier (FBMC), and universal filtered multicarrier (UFMC)) in terms of ranging accuracy, where they seek for very accurate positioning even in rich-scattered NLOS fading channels. They used an energy detector with dynamic threshold which has low-computational complexity for this evaluation and provided a good ranging accuracy and not an excellent accuracy as pertain in correlation detector. They discussed these waveform candidates for vehicle positioning and device-to-device (D2D) communications in 5G NR cellular networks. As discussed in [14], the ultimate navigation solution should be an integration of different methods, since none of the proposed systems in the literature and implementation guarantee $99.99 \%$ availability in any location under any circumstances.

\section{B. Detect-and-Avoid Systems}

One criterion for integration of UAS into urban and nearurban airspace is to use ground-based DAA for routine UAS terminal area operations. This can be implemented not only on the ground but also with on-board support. NASA [20] used Ikhana aircraft equipped with a prototype DAA system doing 11 flights and 200 scripted encounters with other aircraft. The aircraft was equipped with DAA sensor/radar, ADS-B and traffic alert and collision avoidance system (TCAS). UAM operations are planned to be autonomous, therefore, in a designated UAM operation system, the DAA process cannot be detection only. The central management system should be aware of UAS current and future flight paths, while cooperative sharing of data through on-board sensing hardware that tracks other UAS within range is complementary is also required. UAM central management unit will find the best route for each UAS, which result in solving an optimization problem.

An autonomous central management unit should process data and make decisions accordingly. An adaptive predictive control technique based on fuzzy logic was introduced in [20] for the supervisory layer. The authors of [21] proposed a fast dynamic mixed integer linear programming (MILP) optimization problem for efficient path planning of UAVs in various flight formations. The optimization problem in this paper relies on ADS-B information and estimation of other aircraft velocity/acceleration to minimize time and energy consumption for collision-free formation flight. The solution to the problem is based on a branch and bound technique for two fixed and flexible aircraft formations.

An instance of UAM traffic management scenario is illustrated in Fig. 33, where two UAVs (or vehicles for UAM) want to reach their destinations (i.e., pink and green destinations) with minimum cost by moving along the paths between fueling stations is illustrated. We can represent this scenario as a graph where the vertices (or nodes) of the graph correspond to fueling stations. The UAV routing scheme can be modeled as shortest path problem in graph theory. The aim is to find the minimal total cost of a tour between a starting node and destination node such that each node is visited only once. Let $G=(V, A)$ be a directed graph, where $A$ is the set of all arcs and $V$ is the set of all nodes. Let $\delta^{+}(i)$ and

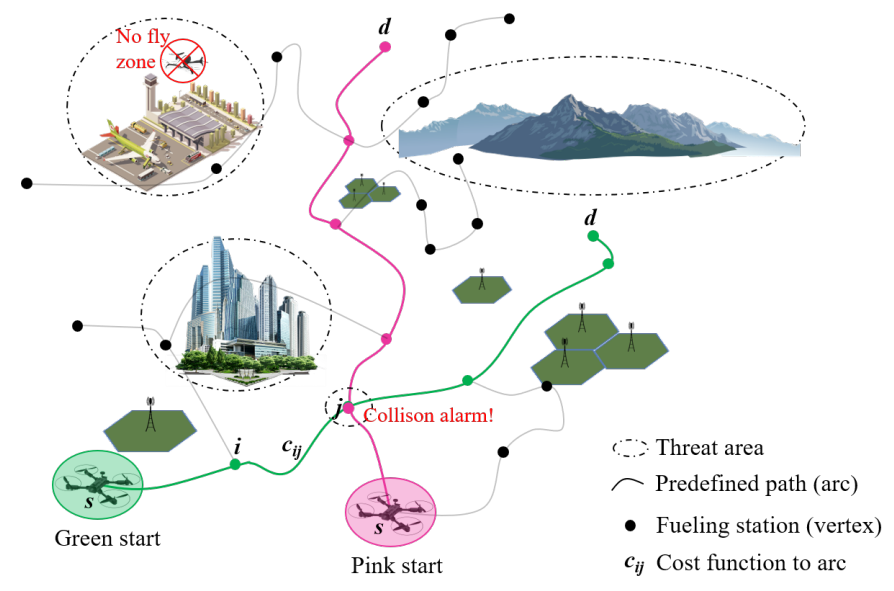

Figure 3. UAM flight trajectory problem: The minimization of the cost (which is a function of reliability of navigation, the risk associated with collision, the range of threat areas, and the location of fueling stations) for each UAV is related to the shortest path problem and TSP depending on the application.

$\delta^{-}(i)$ denote a set of outgoing and incoming arcs of node $i$, respectively. In addition, let $\operatorname{arc}(i, j)$ be the directed path from (i)th vertex to $(j)$ th vertex for $i, j \in V$. Let $c_{i j}$ be the cost associated with $\operatorname{arc}(i, j)$ represented as $\left(X_{i j}\right)$. In general, $c_{i j}$ is a function of reliability of navigation, the risk associated with collision (e.g., two UAVs reach to the same fueling station at the same time), and the range of threat areas (e.g., buildings, mountains, airports, or other no-fly zones, etc.). Let $T_{s, d}$ be a sequence defined as $\left(\operatorname{arc}\left(s, n_{1}\right), \operatorname{arc}\left(n_{1}, n_{2}\right), \ldots,\left(n_{M}, d\right)\right)$ for $n_{k=1,2, \ldots, M}, s, d \in V$, which represents a path (i.e., a tour) on the graph between the vertex $s$ and the vertex $d$. Assuming that $c_{i j}$ does not change in time, from a single UAV (located at the starting vertex $s$ and heading to the destination vertex $d$ ) perspective, The corresponding optimization problem to find the optimum path from node $s$ to node $t$ can be given as follows:

$$
\begin{aligned}
& \tilde{T}_{s, d}=\arg \min _{T_{s, d}} \sum_{(i, j) \in A} c_{i j} X_{i j} \\
& \text { s.t., } \\
& \sum_{(i, j) \in \delta^{+}(i)} X_{i j}-\sum_{(i, j) \in \delta^{-}(i)} X_{j i}=\left\{\begin{array}{ll}
1, & \text { if } i=s \\
-1, & \text { if } i=d \quad \\
0, & \text { otherwise }
\end{array} \quad \forall i \in V\right. \\
& \sum_{(i, j) \in \delta^{+}(i)} X_{i j} \leq 1 \forall i \in V
\end{aligned}
$$

The first constraint is a flow conservation constraint which shows that the sum of the flow through arcs directed toward a node is equal to the sum of the flow through arcs directed away from that node. The second constraint ensures that the outgoing degree of each node be one at most.

In some applications such as mail delivery application where aircraft visit each station exactly once, the problem transform to Hamiltonian path, where the corresponding optimization problem can be modeled as a asymmetric traveling sales- 
man problem (ATSP). The ATSP and is Non-deterministic Polynomial-time hardness (NP-hard). The reason behind this model similarity and our scenario is the very fuel dependent characteristics of UAVs. Therefore, each depot can be a fuel/re-charge station while a designated mission can be accomplished. The UAV starts at a depot and visits a set of targets. A subset of targets can also be considered and therefore, no solution can be presented for the ATSP in the literature, and only heuristic algorithms have been presented thus far [22], [23], [24]. The presented problem here is shown in 2D trajectories, whereas a novel ground-based aircraft separation systems are designed to follow $3 \mathrm{D}$ precision trajectory clearances based on navigation performance requirements (similar to Fig. 3) for manned aircraft. Similar optimization modeling can be exploited for future UAM operations. The corresponding linear integer programming formulation for ATSP can be given by

$$
\begin{aligned}
& \tilde{T}=\arg \min _{T} \sum_{(i, j) \in A} c_{i j} X_{i j} \\
& \text { s.t., } \sum_{(i, j) \in \delta^{+}(i)} X_{i j}=\sum_{(i, j) \in \delta^{-}(i)} X_{i j}=1,
\end{aligned}
$$

where

$$
X_{i j}=\left\{\begin{array}{ll}
1, & \operatorname{arc}(i, j) \in \tilde{T} \\
0, & \text { otherwise }
\end{array} .\right.
$$

The constraint (3) imposes that in-degree and out-degree of each vertex are equal to one. Solving this optimization problem attains the optimum sequence $\tilde{T}_{s, d}$ which minimizes the total cost. There are several methods to solve the ATSP problem in (1) with constraints in (3). One solution is provided in [25] by adopting Lin-Kernighan-Helsgaun heuristic (i.e., one of the common approaches for solving the symmetric TSP problem by swapping pairs of sub-tours to make a new tour) for transformed ATSP. They first transformed the heterogeneous, multiple depot, multiple UAV routing problem-which is a set of heterogeneous UAVs that fly from depots with a set of targets in a sequence, pass once and return to their initial depot after visiting the targets-into a single ATSP using a NoonBean transformation which transform an instance of generalized TSP to an equivalent instance of ATSP. They showed that solutions whose costs are within $16 \%$ of the optimum can be obtained relatively fast for routing problem of 10 UAVs and 40 targets. The authors in [26] presented UAS modeling and simulation results for Houston metroplex (city with two or more major airports and a complex airspace) conducted under FAA system safety management transformation that includes DAA versus see and avoid in different mission profiles, with stochastic parameters including positional variance, fuzzy conflicts, and deviation from planned or intended profiles. They modeled Houston Metroplex by using 24 hours of radar-track data from National Offload Program recorded from the Offload Extractor of the Sector Design and Analysis Tool. The under test DAA system modeled as conflict detection every 2 minutes with a look ahead range of 8 minutes. The look ahead covered all the flights in the sector using their planned/current $4 \mathrm{D}$ profile. When conflict was predicted with any other manned flight, the resolution logic was only applied to the UAS and not to the manned flight. The conflict avoidance procedure starts with a speed reduction, a vector to avoid the conflict zone and then return to the original profile, and finally a lateral offset to the original planned profile until top of descent.

\section{Detect-and-Avoid Using Chirp Signals}

Sense and avoid for small-UAS with size, weight and power (SWAP) requirements was discussed in [27]. Their sense and avoid radar system was designed to be a multi channel, frequency modulated continuous wave (FMCW) or chirp radar. Chirp systems simultaneously transmit swept frequency signals and receive target echos. Similar approaches were used for channel characterization using chirp signals in [28] except that the transmitter and receiver can be located at different coordinates. As described in [27] and [28], windowing and fast-Fourier transforms are performed to obtain range (by estimating the gains of the multi-path components) and speed (by estimating Doppler shift) of the target. One challenge in DAA chirp systems is dealing with the leakage of signal resulting from the LOS radiating path. Authors in [27] used Analog Devices integrated circuits (i.e., ADF4158 and AD8283) for radar applications, which provide 6 channel analog-to-digital converters in their modified evaluation board. This solution was motivated by cost and safety requirements for small UAVs for the future.

New autonomous vehicles are using advanced driver assistance system (ADAS) with forward-collision warning tool. These systems enable semi-self driving cars by using sensors such as light, imaging detection, and ranging (LIDAR), camera and radar. FMCW radars being used in ground vehicle's DAA systems and can be a good candidate for future UAV implementation. The authors in [29] investigated FMCW radar-toradar interference, which impose a ghost-target problem in cars. They proposed different chirp slope in time-frequency (TF) domain to reduce interference. Implementing these sensors for scenarios with high UAV-to-UAV collision probability enabled by novel interference mitigation techniques is suitable. In [30], chirp signals are synthesized by using Bessel functions and Fresnel integrals as frequency-domain spectral shaping for DFT-s-OFDM with the motivation of standard-compliance and dual radar-and-communication function, which may also be important features for UAM.

\section{6- LEO Satellite Constellations}

Advancements in reusable rocket technologies, solar cells, ion propulsion systems, and spacecraft collision avoidance systems together with precise navigation systems, phased array antenna systems and high bandwidth communication strategies open up a new era in SWAP LEO constellation ( $250 \mathrm{~kg}$ weight and $600 \mathrm{~km}$ altitude) communication architectures. As the two way communication (from GS to LEO satellite $(600 \mathrm{~km})$ and back to GS) propagation delay is about $4 \mathrm{~ms}$, this architecture enables delay sensitive, high data rate applications that were impractical with a medium-earth orbit (MEO) (2000 km alti- 


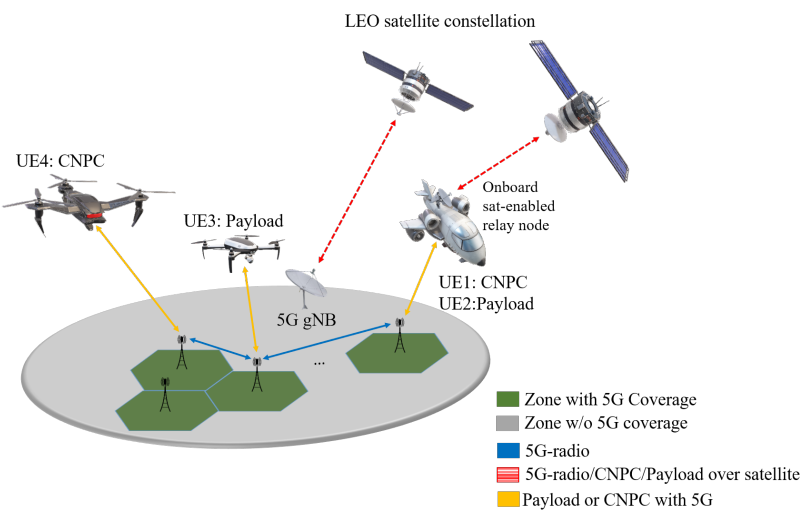

Figure 4. BLOS CNPC link using the $5 \mathrm{G}$ relay node concept: on-board $5 \mathrm{G}$ relay node and LEO satellite [14]

tude) / geosynchronous-earth orbit (GEO) (36000 km altitude) satellites (where two way propagation delays varies between $100 \mathrm{~ms}$ to $250 \mathrm{~ms})^{1}$.

There are many projects targeting this service utilizing LEO satellites: Starlink of SpaceX [31], oneWeb, Facebook (PointView's Athena) [32], and Kuiper of Amazon [33]. Among those, Starlink is targeting more number of satellites in the Northern U.S. and Canada in 2020, rapidly expanding to near global coverage of the populated world by 2021. Other projects have a plan for starting their services before 2025 . When these schedules are projected on a UAM timeline, there is a possibility that LEO constellations could support CNS technologies towards UAM applications. Robustness of the technologies should be evaluated from UAM requirements perspective as they begin to take shape in the upcoming years.

The upcoming LEO satellite providers are focusing on providing data links, not just to ground users, but also to serve as the backbone for remote cellular towers, where cost of installing ground cables is prohibitive. These system may address or compliment UAM communications when the LOS link is not available. Even though the propagation delay for LEOs is significant compared to that of terrestrial links, a single LEO satellite can provide a single-hop link between two points providing a footprint coverage of several hundred $\mathrm{km}$.

A possible approach to smoothly integrate UAV into the $5 \mathrm{G}$ systems via BLOS links using LEO satellites is the relay networks illustrated in Fig. 4 A low-complexity satelliteenabled relay node $(\mathrm{RN})$ onboard a UAV transports the $5 \mathrm{G}$ DL/UL waveform via a LEO link to the terrestrial base station, the so-called donor NodeB at the ground earth station. For the on-board equipment (flight controller requiring CNPC or payload equipment requiring a high-throughput link) the RN appears like a GS. The donor NodeB, for whom the RN is transparent, simply sees a number of users. While this approach may not be optimal in the sense of achieving channel capacity, it requires less communication infrastructure both on-

\footnotetext{
${ }^{1}$ These values only consider the propagation delay, there will be additional processing and application delays.
}

board in UAM node and at the GS, simplifies handover from $5 \mathrm{G}$ to satellite, and leverages $5 \mathrm{G}$ technology.

\section{7- Conclusions}

UAM will provide a new era for aeronautical CNS. Critical UAM requirements are derived from C2 (particularly in RPIC scenarios), data connectivity for passengers and critical flight systems, UAS-to-UAS communication to avoid collision, and data exchange for positioning and surveillance.

Future challenges for UAM operations are predicted to be safety and integration barriers across the entire UAM ecosystem in highly populated zones; accurate and navigation and surveillance capabilities; interactions between vehicles for both traditional and novel UAM airspace management systems; DAA capabilities; handling the loss of primary communications; and public acceptance or response to vehicles. These all represent areas of future investigation. Specifically, the following issues has to be addressed extensively.

- The requirements for robust UAM scenarios will have many updates during the development stages. To have a successful operation of UAM, requirements to possible solutions must be addressed systematically.

- Quantification of the integrity, availability, security, and robustness/reliability is a key enabler of UAM.

- 5G studies and working groups are solely based on cellular companies and vendors. Requirements of aviation support must be addressed in 5G standards including UAM applications.

- Dense and highly obstacle-laden UAM environments will require diverse navigation and detect \& avoid systems compared to commercial aircraft scenarios: fusing (with extensive tracking capabilities) GNSS, Inertial Measurement Unit (IMU), radar(s), Satcom, collisionavoidance/surveillance broadcast information (generally studied under RTCA), and 5G data communications to address these issues should be further investigated.

- The success of UAM will depend on the existence of practically-implementable-theoretically-provable solutions. High-complexity, real-time CNS applications in a "Moores-law approaching to bound" era will lead us to implementable yet sub-optimal solutions.

\section{REFERENCES}

[1] NASA, "Urban Air Mobility (UAM) Market Study," Oct. 2019. [Online]. Available: https://www.nasa.gov/sites/default/files/atoms/files/ uam-market-study-executive-summary-v2.pdf

[2] FAA, "UAS remote identification," 2020, last accessed 21 January 2020. [Online]. Available: https://www.faa.gov/uas/research_ development/remote_id/

[3] - "UAS identification and tracking aviation rulemaking committee (ARC)," 2017, last accessed 04 March 2020. [Online]. Available: https://www.faa.gov/regulations_policies/rulemaking/committees/ documents/media/UAS\%20ID\%20ARC\%20Final\%20Report\%20with\% 20Appendices.pdf

[4] —_, "Traffic management overview," 2016, last accessed 04 March 2020. [Online]. Available: https://www.faa.gov/nextgen/programs/ weather/tfm_support/overview/ 
[5] RTCA, "Command and control (C2) data link minimum operational performance standards (MOPS)-terrestrial," Radio Technical Commission for Aeronautics, May. 2016.

[6] ITU, "Compatibility study to support the line-of sight control and nonpayload communications link(s) for unmanned aircraft systems proposed in the frequency band 5030-5091 mhz," ITU-R M.2237, Nov. 2011.

[7] 3GPP, "Study on New Radio (NR) to support non-terrestrial networks," TS 38.811 v15.2.0, Oct. 2019.

[8] — "Physical channels and modulation (Release 16)," TS 38.811 V16.0.0, Jan. 2020.

[9] _ "Solutions for NR to support non-terrestrial networks (NTN)," TR 38.821 v16.0.0, Jan. 2020.

[10] — "LTE physical layer; General description (Release 15)," 3GPP TS 36.201 V15.1.0, pp. 1-14, Jan. 2018.

[11] Inmarsat, Intelsat, SES, and Fraunhofer IIS, "OFDM operation over satellite," RP-192203, Sep. 2019.

[12] — "OFDM operation over satellite," RP-192237, Sep. 2019.

[13] J. Poss, "Shouldn't we all squawk when flying?"," Inside Unmanned System Magazine, pp. 20-22, Jan. 2020.

[14] N. Hosseini, H. Jamal, J. Haque, T. Magesacher, and D. W. Matolak, "UAV command and control, navigation and surveillance: A review of potential 5G and satellite systems," in Proc. IEEE Aerospace Conference, Mar. 2019, pp. 1-10.

[15] D. Shibasaki, "Low cost GNSS receiver system for high precision GNSS data processing," 2019, last accessed 5 October 2019. [Online]. Available: http://www.unoosa.org/documents/pdf/psa/ activities/2019/UN_Fiji_2019/S2-10.pdf

[16] Huawei, "5G based high accuracy positioning," 2019, last accessed 5 October 2019. [Online]. Available: https://legacy.viestintavirasto.fi/ attachments/esitykset/5G2015_Huawei.pdf

[17] F. Peirong, L. Wenyi, C. Xiaowei, and L. Mingquan, "RTK with the assistance of an IMU-based pedestrian navigation algorithm for smartphones," Sensors, vol. 19, no. 16, p. 3586, 2019.

[18] D. Peral-Rosado, A. José, J. Saloranta, G. Destino, J. A. López-Salcedo, and G. Seco-Granados, "Methodology for simulating 5G and GNSS high-accuracy positioning," Sensors, vol. 18, no. 10, p. 3220, 2018.

[19] X. Cui, T. A. Gulliver, J. Li, and H. Zhang, "Vehicle positioning using 5G millimeter-wave systems," IEEE Access, vol. 4, pp. 6964-6973, 2016.

[20] P. Merlin and J. Banke, "NASA, industry complete third phase of UAS flight testing," 2019, last accessed 5 October 2019. [Online]. Available: https://www.nasa.gov/centers/armstrong/features/detect_and_avoid.html
[21] R. Asep, A. Achaibou, and F. Mora-Camino, "Automatic collision avoidance based on supervised predictive controllers," Control Engineering Practice, vol. 4, no. 8, pp. 1169-1175, 1996.

[22] H. Kaplan, M. Lewenstein, N. Shafrir, and M. Sviridenko, "Approximation algorithms for asymmetric TSP by decomposing directed regular multigraphs," Journal of the ACM (JACM), vol. 52, no. 4, pp. 602-626, 2005.

[23] M. Bläser, "A new approximation algorithm for the asymmetric TSP with triangle inequality," ACM Trans. Algorithms, vol. 4, no. 4, Aug. 2008.

[24] S. Khuller, A. Malekian, and J. Mestre, "To fill or not to fill: The gas station problem," ACM Trans. Algorithms, vol. 7, no. 3, Jul. 2011.

[25] P. Oberlin, S. Rathinam, and S. Darbha, "Today's traveling salesman problem," IEEE Robotics Automation Magazine, vol. 17, no. 4, pp. 7077, Dec. 2010.

[26] S. S. Borener, D. Hufty, V. S. Guzhva, K. Martin, and R. Fraga, "Modeling emergent risks in complex airspace: UAS operations in a metroplex environment," in Proc. IEEE/AIAA Digital Avionics Systems Conference (DASC), Sep. 2015, pp. 5B3-1-5B3-13.

[27] Lei Shi, C. Allen, M. Ewing, S. Keshmiri, M. Zakharov, F. Florencio, N. Niakan, and R. Knight, "Multichannel sense-and-avoid radar for small UAVs," in Proc. IEEE/AIAA Digital Avionics Systems Conference (DASC), Oct. 2013, pp. 6E2-1-6E2-10.

[28] N. Hosseini and D. W. Matolak, "Wide band channel characterization for low altitude unmanned aerial system communication using software defined radios," in Proc. IEEE Integrated Communications, Navigation, Surveillance Conference (ICNS), Apr. 2018, pp. 2C2-1-2C2-9.

[29] Y. S. Son, H. K. Sung, and S. W. Heo, "Automotive Frequency Modulated Continuous Wave Radar Interference Reduction Using PerVehicle Chirp Sequences," Sensors, vol. 18, no. 9, p. 2831, 2018.

[30] A. Şahin, H. Hosseini, H. Jamal, and D. Matolak, "DFT-spread-OFDM based chirp transmission," in Proc. IEEE Wireless and Microwave Technology Conference (WAMICON), Apr. 2020, pp. 1-5.

[31] J. Foust, "Spacex's space-internet woes: Despite technical glitches, the company plans to launch the first of nearly 12,000 satellites in 2019,' IEEE Spectrum, vol. 56, no. 1, pp. 50-51, Jan. 2019.

[32] M. Harris, "Tech giants race to build orbital internet [news]," IEEE Spectrum, vol. 55, no. 6, pp. 10-11, Jun. 2018.

[33] Caleb Henry, "Amazon moving Project Kuiper team to new R\&D headquarters," 2019, SPACENEWS. [Online]. Available: https://spacenews. com/amazon-moving-project-kuiper-team-to-new-rd-headquarters/ 